\title{
An Application of Hadamard Transform for Plasma Laser-Induced Fluorescence Spectroscopy
}

\author{
Hiroyuki ARAKAWA, Shigeru INAGAKI ${ }^{1,2)}$, Makoto SASAKI ${ }^{1,2)}$, Kimitaka ITOH $^{2,3)}$ \\ and Sanae.-I. ITOH ${ }^{1,2)}$ \\ Teikyo University, 6-22 Misakimachi, Omuta 836-8505, Japan \\ ${ }^{1)}$ Research Institute for Applied Mechanics, Kyushu University, 6-1 Kasuga-Koen, Kasuga 816-8580, Japan \\ ${ }^{2)}$ Research Center for Plasma Turbulence, Kyushu University, 6-1 Kasuga-Koen, Kasuga 816-8580, Japan \\ 3) National Institute for Fusion Science, 322-6 Oroshi-cho, Toki 502-5292, Japan
}

(Received 22 August 2015 / Accepted 30 September 2015)

\begin{abstract}
An application of Hadamard transform method to laser-induced fluorescence spectroscopy (LIF) is proposed, in order to improve spatial resolution, signal-to-noise ratio, and experimental efficiency. The new method allows for measurement of spatial structure of ion flow and/or temperature efficiently with single detector by changing Hadamard mask configurations.
\end{abstract}

(c) 2015 The Japan Society of Plasma Science and Nuclear Fusion Research

Keywords: Hadamard transform, laser-induced fluorescence, Doppler shift spectroscopy, absolute velocity measurement, absolute temperature measurement

DOI: $10.1585 /$ pfr. 10.1201085

The importance of measurements of inhomogeneities in plasma is widely recognized to understand the selforganization mechanisms in magnetized plasmas and to improve a quality of products processed by industrial plasmas. There is variety of plasma parameters should be measured. Some diagnostic tools for plasma parameter, e.g. spectroscopy of weak light, have a low signal-to-noise ratio (SNR). A conditional averaging (or lock-in amplifier) method is very powerful to improve SNR if the noise is random. However, a long averaging time is necessary to increase the SNR. To improve the SNR tenfold, a hundredfold averaging time is required. It severely impairs the experimental efficiency to observe the spatial structures by scanning of single detector. In optical measurement, an efficient conditional averaging scheme based on the Hadamard transform method [1], which uses ordered masks either block or transmit light, is frequently used. Here we propose an application of the Hadamard transform method to plasma diagnostics. As a specific diagnostic, we considered the laser-induced fluorescence spectroscopy (LIF) $[2,3]$, which can derive local absolute ion flow velocity and ion temperature with velocity distribution measurements. Our new method is named as Hadamard laserinduced fluorescence (HLIF). At the spatial structure measurement, conventional LIF methods require realignment of incident laser beam and fluorescence collection optics mechanically, in addition to the laser frequency scan. Thus, the spatial and reproducibility resolution is at most a few mm [4]. Using PMT array detector is a method to increase in the reproducibility resolution. However, the number of spatial measure points is limited by the number of

author'se-mail: arakawa@fmt.teikyo-u.ac.jp detectors. In the HLIF method, we can easily change the number of spatial measure points. Depending of the conditions, there is a possibility of improving spatial resolution with single detector. The method proposed here optimizes the SNR and the spatial resolution.

An application of the HLIF method in linear magnetized plasma, such as for LMD-U and PANTA [5], is considered. The device has cylindrical helicon plasma with a diameter of approximately $0.1 \mathrm{~m}$ and an axial length of $\sim 4 \mathrm{~m}$ is produced by the $\mathrm{RF}$ wave $(7 \mathrm{MHz}, 3 \mathrm{~kW})$ in a quartz tube and is radially restricted by the axial magnetic field. Typical plasma parameters are, $3 \mathrm{eV}$ electron temperature and $10^{18} \mathrm{~m}^{-3}$ electron density at the operational conditions of 2 mTorr argon gas pressure and $900 \mathrm{G}$ magnetic field strength. The HLIF method adds Hadamard mask to the collection optics of fluorescence induced by laser beam. The components of this system are frequency tunable laser, laser beam collimation optics, optical collection optics, a detector, data acquisition system and a series of Hadamard mask, respectively. Schematic views of radial, azimuthal and axial ion flow profile measurement are shown in Fig. 1. Each system can also measure ion temperature profile by spectral broadening of fluorescence. Here, we consider a measurement of the radial ion temperature profile which has $m$ split elements by showing Fig. 1 (a). At the $j$-th frequency of laser, emitted light from the fluorescence line, $\psi_{m j}$, is focused on to the mask. The radiation passing through the $k$-th Hadamard mask is collected by single detector, and the strength of this signal, $\eta_{k j}$, is recorded. After the radiation has been measured, the mask configuration is replaced by another, and the procedure repeated until the $m$-th mask measurements have been made 
(a)
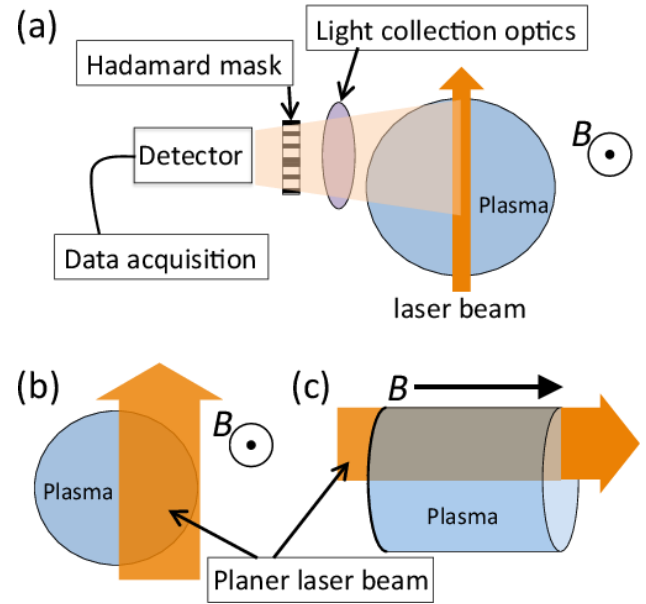

Fig. 1 Schematic views of (a) radial, (b) azimuthal and (c) axial ion flow profile measurement of Hadamard laser-induced fluorescence. Each system can also measure ion temperature profile by spectral broadening. The collection system is also shown in (a).

with each laser frequency. The detected signals, $\eta_{m j}$, with $m$ masks at $j$-th frequency of laser can be represented as,

$$
\eta_{m j}=S_{m} \psi_{m j}
$$

where, $S_{m}$ is the S-matrix which is ordered binary matrix and mathematical representation of a series of the Hadamard mask. The construction methods of S-matrices with the orders of $m=2^{M}-1, M=1,2,3 \cdots$ is described in textbook [1]. An example of S-matrix at $m=7$ is shown as,

$$
S_{7}=\left[\begin{array}{lllllll}
1 & 0 & 1 & 0 & 1 & 0 & 1 \\
0 & 1 & 1 & 0 & 0 & 1 & 1 \\
1 & 1 & 0 & 0 & 1 & 1 & 0 \\
0 & 0 & 0 & 1 & 1 & 1 & 1 \\
1 & 0 & 1 & 1 & 0 & 1 & 0 \\
0 & 1 & 1 & 1 & 1 & 0 & 0 \\
1 & 1 & 0 & 1 & 0 & 0 & 1
\end{array}\right] .
$$

The Hadamard mask is ordered with each row (or column) of S-matrix and is constructed with open and closed slots. In the S-matrix, 1 is the open and 0 is the closed mask. An example of the series of one-dimensional (profile) masks of ordered with $S_{7}$ is shown in Fig. 2. If we requires the spatial resolution less than $1 \mathrm{~mm}, \mathrm{~S}$-matrix orders of $m=63$ is required as the radius of the PANTA is $\sim 5 \mathrm{~cm}$. The Hadamard transform is one of a orthogonal transform. Therefore, the emitted intensity profile at the $j$ th frequency of laser can be obtained by using the inverse of S-matrix as,

$$
\psi_{m j}=S_{m}^{-1} \eta_{m j} .
$$

This procedure repeats through the frequency scan of laser; the scan frequencies are around the excitation line of targeted ions or neutrals. After that, velocity distribution of

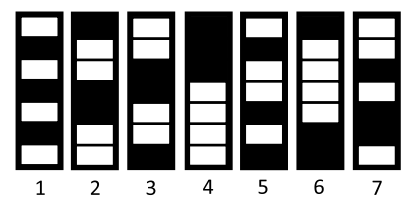

Fig. 2 The series of one dimensional Hadamard mask of $m=7$.

each local position is revealed. The each local ion flow velocity or ion temperature is obtained by the Doppler shift or Doppler broadening of each velocity distribution.

The improvement of SNR compared with conventional LIF ( $\left.I_{\mathrm{SNR}}\right)$ at the $m$ divided spatial distribution case is estimated as [1],

$$
I_{\mathrm{SNR}} \propto \frac{m+1}{2 \sqrt{m}} \approx \frac{\sqrt{m}}{2} .
$$

From another perspective, at the same SNR condition, the statistics can be decreased with the factor of $\approx 4 /(m+2)$. Here, we suppose the intensity and the background noise of fluorescence at each position are considered same as HLIF and LIF. We should note that in the case of Figs. 1(b) and (c), SNR may not increased compared with conventional LIF as these require sheet shaped laser beam and the beam intensity at a local position is decreased. Nevertheless, our method is an efficient method rather than conventional method as the conventional one requires frequency scan at each position and optical realignment for changing its detection position. Our method requires a series of frequency scan and replacement of masks without realign the position of optics and laser. We should also note that the improvement of spatial resolution of the HLIF method compared with the conventional method should be tested in experiments. Because the effective spatial resolution is restricted by not only the masks configuration (size) but also the masks manufacturing accuracy and replacement accuracy of the masks.

In conclusion, we have proposed the new optics based on the Hadamard transform for plasma diagnostics and applied this method to the laser-induced fluorescence spectroscopy. The new optics can measure ion velocity distribution function without mechanical realignment, but with changing Hadamard mask configurations. The SNR of $m$ measure points is increased by $\sqrt{m} / 2$ compared with the conventional LIF method. The Hadamard transform method is very flexible and possible to be applied to the other diagnostics for plasma parameters.

This work is supported by the grant-in-aid for Scientific Research of JSPS (15H02155, 15H02335), and by Asada Science Foundation.

[1] M. Harwit and N.J. Sloane, Hadamard Transform Optics (Academic Press, London, 1979).

[2] R.A. Stern and J.A. Johnson, Phys. Rev. Lett. 34, 1548 (1975). 
[3] F. Anderegg, R.A. Stern, F. Skiff, B.A. Hammel, M.Q. Tran, P.J. Paris and P. Kohler, Phys. Rev. Lett. 57, 329 (1986).

[4] E. Scime, R. Hardin, C. Biloiu, A.M. Keesee and X. Sun, Phys. Plasmas 14, 043505 (2007).
[5] H. Arakawa, S. Inagaki, Y. Nagashima, T. Yamada, K. Kamataki, T. Kobayashi, S. Sugita, M. Yagi, N. Kasuya, A. Fujisawa, S.-I. Itoh and K. Itoh, Plasma Phys. Control. Fusion 52, 105009 (2010). 